\title{
A Weak Form Nonlinear Model for Thermal Sensitivity of Love Wave Mode on Layered Structures
}

\author{
Yang Yang, Harshad Mishra, Student Member, IEEE, Qiaozhen Zhang, Sami Hage-Ali, Member, IEEE, \\ Tao Han*, Member, IEEE, and Omar Elmazria*, Senior Member, IEEE
}

\begin{abstract}
A precise theoretical model for the thermal sensitivity of Love wave mode is significant in the structure design, temperature compensation, and the prediction of thermal behavior. This paper proposes a weak form nonlinear model to calculate the thermal sensitivity of Love waves on arbitrary layered structures. The third-order material constants, as well as the thermal stress and strain tensors between the substrate, electrodes, and wave-guiding layer, are taken into account in the model. The $9 \times 9$ effective elastic matrix and the $3 \times 9$ effective piezoelectric matrix are imported into the nonlinear constitutive equations and boundary conditions using weak form expressions. A temperature-compensated Love wave mode resonator on a layered $\mathrm{ZnO} / \mathrm{IDT} / q u a r t z$ structure is obtained. The theoretical model is verified through the comparison of experimental and analytical results. The model is beneficial for the design of Love wave devices and sensors.
\end{abstract}

Index Terms - Love wave; third-order constants; frequency temperature characteristic; thermal sensitivity

\section{INTRODUCTION}

$\mathrm{S}$ Ingle-port Love wave mode sensors have the promising potential of being wireless sensing platforms for measuring physical or chemical parameters, such as gas and liquid sensing [1], [2]. Love waves are shear waves that propagate in a layered structure consisting of a substrate and a film layer. The film acts as a wave-guiding layer deposited on the substrate. The condition for the Love wave mode is that the shear velocity of the wave-guiding layer is smaller than that of the substrate. The substrate can be quartz, $\mathrm{LiNbO}_{3}$, $\mathrm{LiTaO}_{3}$ or other piezoelectric materials. On one hand, the sensitive film is deposited directly on the wave-guiding layer as an active sensing layer, which enhances the sensing areas and thus improves the sensitivity to surface disturbances. On the other hand, the interdigital transducer (IDT) and reflector

Manuscript received July 29, 2019. This research was supported by the National Key Research and Development Program of China (2016YFB0402700), National Natural Science Foundation of China (under grant No. U1837210, No.11774230 and No.61531008), Key Research and Development Program of Jiangsu Province (Grant No. BE2018008-5), and National Natural Science Youth Foundation of China (Grant No. 11904233). (*Corresponding author: Tao Han, Omar Elmazria.)

Yang Yang, and Tao Han are with the School of Electronic Information and Electrical Engineering, Shanghai Jiao Tong University, Shanghai 200240, China. (e-mail: yangyang2015@ sjtu.edu.cn; than@ sjtu.edu.cn)

Harshad Mishra, Sami Hage-Ali, and Omar Elmazria are with the Institute Jean Lamour UMR 7198, Université de Lorraine - CNRS, Nancy, France. (email: omar.elmazria@univ-lorraine.fr)

Qiaozhen Zhang is with the School of Information, Mechanical and Electrical Engineering, Shanghai Normal University, Shanghai 200234, China. (e-mail: zhangqz@shnu.edu.cn) gratings are naturally protected by the insulating waveguiding layer [3]. However, the frequency fluctuation of the Love wave mode caused by the environmental temperature is a crucial factor affecting the measurement performance. In order to accurately predict the thermal behavior, design a new structure of Love wave mode devices, and optimize the temperature compensation, a precise theoretical model for the thermal sensitivity of Love wave mode attracts much attention.

Apart from the experimental studies on the thermal sensitivities of Love waves on quartz substrates [4]-[6], several theoretical models have been investigated. Jakoby et al. have numerically analyzed the rotated quartz cuts and guiding layer thicknesses for Love wave devices using the propagator matrix approach, and a zero temperature coefficient of frequency (TCF) Love wave device at $35{ }^{\circ} \mathrm{C}$ is obtained [7], [8]. Josse has derived the effective permittivity function of the film-substrate interface to study the frequencytemperature characteristics of Love wave devices without considering the internal stress of the substrate by the overlay. The frequency variation of $80 \mathrm{ppm}$ is measured over a temperature range $-30{ }^{\circ} \mathrm{C}$ to $40{ }^{\circ} \mathrm{C}$, which is greater than the shift predicted by theoretical calculations [9]. The frequencytemperature characteristics of Love wave mode on quartz have been investigated using the numerical Campbell and Jones method [10], [11]. The frequency shift in the temperature range from $20^{\circ} \mathrm{C}$ to $80^{\circ} \mathrm{C}$ is $170 \mathrm{ppm}$, and the zero TCF is obtained at $25^{\circ} \mathrm{C}$ [11]. A finite element analysis/ boundary element method (FEA/BEM) has been investigated for the thermal sensitivity based on a material coefficient perturbation according to Campbell \& Jones approach [10], [12]. The experimental turnover temperature occurs $20{ }^{\circ} \mathrm{C}$ below the theoretical temperature [12]. It is not a general model for the analysis of thermal effects on surface acoustic waves [13].

The perturbation theory is a mathematical method for finding approximate solution to a problem. It is widely used in various areas including the study for the thermal behavior of surface acoustic waves [14]. Sinha and Tiersten have presented the perturbation theory to calculate the temperaturevelocity characteristics for surface acoustic wave resonators [15]. Ballandras et al. have extended the perturbation theory to take into account the influence of metallization on the thermal sensitivity of Rayleigh wave for periodic grooves and metal strip gratings [16]. The thermal stress and strain distributions among the substrate, layer, and electrodes are dependent on temperatures. In a layered structure, the thermal 
expansion of the substrate, the layer, and electrodes is different. The thermal stress and strain induced by the temperature are important and non-negligible in the calculation of the thermal sensitivity. In addition, because of the anisotropy of some substrates and layers, the thermal expansions are also anisotropic. Therefore, the boundary conditions for the calculation of surface acoustic waves are complicated and need to be considered with the thermal stress and strain tensors arising from the differential thermal expansion among the substrate, the layer, and electrodes in layered structures.

The purpose of this paper is to develop a weak form nonlinear model for computing the thermal sensitivity of the Love wave mode on the quartz substrates accurately. This model presented in the paper combines the perturbation theory and the finite element method (FEM) for the thermal sensitivity of the Love wave mode. The FEM solver is utilized to accurately model the thermal stress and strain tensors of the film, substrate, and electrodes, as well as the interfaces between them. The nonlinear constitutive equations and boundary conditions are modified using weak form expressions with the combination of the contribution of thermal stress and strain tensors, as well as the influence of the third-order material constants of the substrate, electrodes, and layers. The thermal properties of Love wave mode on the quartz substrate are studied to verify the model. The calculations of frequency-temperature characteristics match well with the experiment results. By optimizing the thickness of the wave-guiding layer, a temperature-compensated Love wave resonator with an enhanced electromechanical coupling factor is obtained, with the relative frequency shift less than $50 \mathrm{ppm}$ in the temperature range of $25{ }^{\circ} \mathrm{C}$ to $60{ }^{\circ} \mathrm{C}$. This model improves the prediction accuracy of the frequencytemperature characteristics and is beneficial for the design of temperature-compensated Love wave devices.

The remaining of the paper is organized as follows: In section II, the weak form nonlinear model is analyzed for the temperature behavior of Love wave mode. In section III, the accuracy of the model is verified by experiments. Conclusions are discussed in section IV.

\section{MODEL ANALYSIS}

The three-dimensional model of a layered wave-guiding layer/IDT/quartz structure is established, as shown in Fig. 1. The symbol $\mathrm{x}$ is the propagation direction, and $\mathrm{y}$ is the aperture direction. The perfectly matched layer is applied to the bottom of a quartz substrate to reduce the wave reflection at the bottom and the model size. The thermal field, solid mechanical field, and static electric field are added into the model. The mesh is dynamically adaptive to the material deformation.

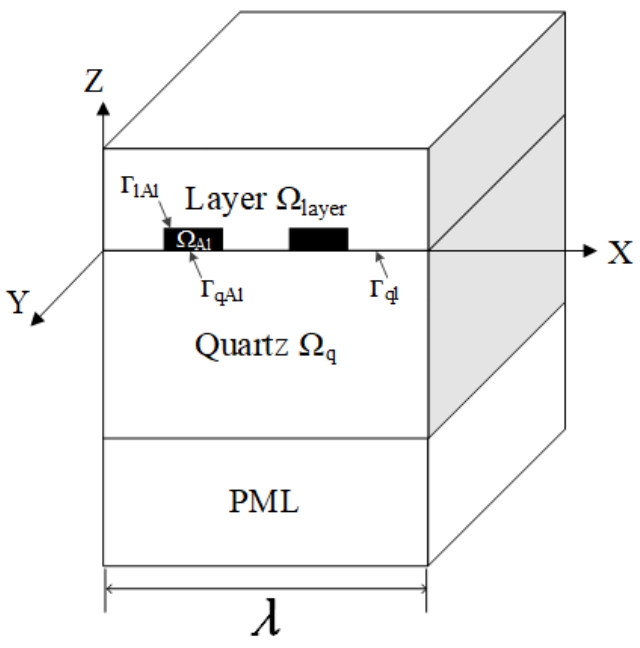

Fig. 1. The three-dimensional layered Love wave structure on quartz.

The thermal sensitivity analysis is based on the weak form expressions in a Lagrange description, as shown in Fig. 2. Firstly, the model is set in a natural state with no deformation at the reference temperature $\Theta_{0}$. Then, a thermal biasing field at the given temperature $\Theta$ is applied in an intermediate state, resulting in the thermal expansion [17]. The thermal stresses and strains mainly concentrate on the interfaces between the electrode, wave-guiding layer, and substrate according to the results of the stationary analysis. Finally, the thermal stress and strain tensors of the structure are superimposed in the final state. The displacement distribution and resonance frequency of the Love wave mode are obtained based on the modal analysis.

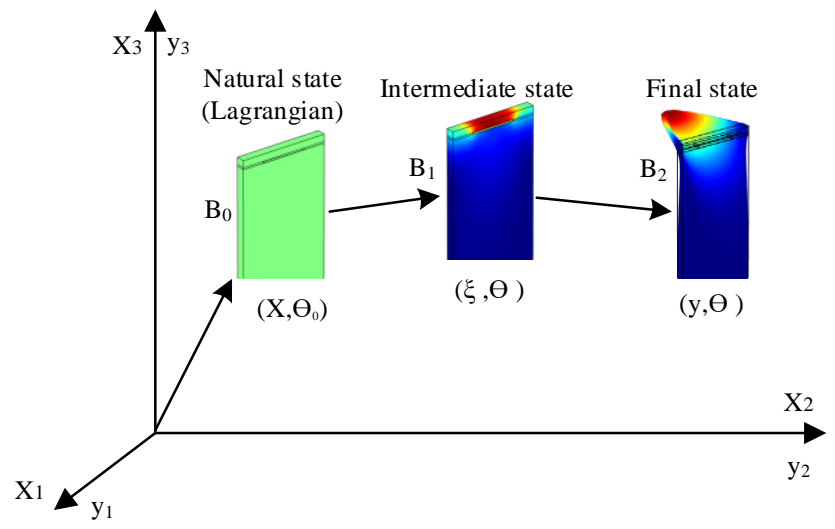

Fig. 2. The schematic diagram of the thermal deformation in Lagrange description.

In the model, the fundamental elastic, piezoelectric, and dielectric constants are replaced by the effective material constants dependent on the thermal biasing field. The thirdorder material constants are imported into effective material constants. At a given temperature $\Theta$, because of the different thermal expansion coefficients of the wave-guiding layer, electrode, and substrate, the thermal strain tensors of mesh nodes are diverse from each other. The boundary conditions of interface surfaces are complicated, which increases the 
difficulty in calculating the thermal sensitivity by solving the nonlinear piezoelectric constitutive equations.

In order to accurately solve the thermal sensitivity of surface acoustic waves on the layered structure in a thermal field, the weak form partial differential equation (PDE) in finite element analysis modifies the nonlinear constitutive equations flexibly using numerical integration of the original form PDE. The mesh nodes are integrated by weak form equations in the solution domain, which reduces the maximum order of the spatial derivatives with the advantage of the fewer continuity boundary condition requirements compared to the original form piezoelectric PDE. The weak form nonlinear piezoelectric constitutive equations are defined as follows:

$$
\left\{\begin{array}{l}
\iiint_{\Omega} \sum_{i=1}^{9}\left(\sum_{j=1}^{9} G_{i j}^{\delta} \nabla u_{j}^{\delta}+\sum_{k=1}^{3} R_{i k}^{\delta} \nabla \varphi_{k}^{\delta}\right) v \mathrm{~d} \Omega=\iiint_{\Omega}\left(T^{\delta}-\bar{T}^{\delta}\right) v \mathrm{~d} \Omega \\
\iiint_{\Omega}^{3} \sum_{i=1}^{3}\left(\sum_{j=1}^{9} R_{i j}^{\delta} \nabla u_{j}^{\delta}-\sum_{k=1}^{3} N_{i k}^{\delta} \nabla \varphi_{k}^{\delta}\right) v \mathrm{~d} \Omega=\iiint_{\Omega} D^{\delta} v \mathrm{~d} \Omega
\end{array}\right.
$$

where $G_{i j}, R_{i k}, N_{i j}$ are the effective elastic constant, effective piezoelectric constant, and effective dielectric constant in Lagrange coordinate, respectively. $\Omega$ denotes the solution domain of the substrate and wave-guiding layer. $\delta$ represents the mesh node in the solution domain. $u_{i}^{\delta}, \varphi_{k}^{\delta}, T^{\delta}, \bar{T}^{\delta}$ and $D^{\delta}$ are the displacement, electric potential, the second PiolaKirchhoff stress tensor, initial stress tensor, and electric displacement of the node, respectively. $v$ is the test function utilizing the Lagrange multiplier.

The effective piezoelectric constants of metal electrodes are set to zero due to non-piezoelectric property. Therefore, the weak form expressions are defined as follows:

$$
\left\{\begin{array}{l}
\iiint_{\Omega} \sum_{i=1}^{9} \sum_{j=1}^{9} G_{i j}^{\delta} \nabla u_{j}^{\delta} v \mathrm{~d} \Omega=\iiint_{\Omega}\left(T^{\delta}-\bar{T}^{\delta}\right) v \mathrm{~d} \Omega \\
\iiint_{\Omega} \sum_{i=1}^{3} \sum_{k=1}^{3} N_{i k}^{\delta} \nabla \varphi_{k}^{\delta} v \mathrm{~d} \Omega=\iiint_{\Omega} D^{\delta} v \mathrm{~d} \Omega
\end{array}\right.
$$

Fig. 3 presents the thermal strain and stress distributions in the meshes. The thermal strains mostly distribute in the electrode. Simultaneously, the thermal stresses primarily distribute in the wave-guiding layer and electrode. The stress and strain distributions of all mesh nodes are integrated using Lagrange interpolation in the solution domain.

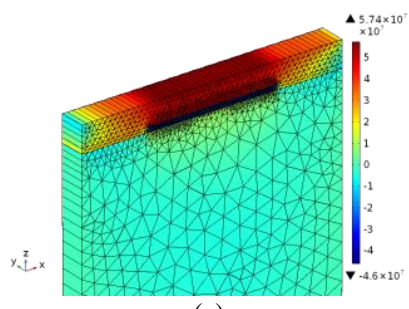

(a)

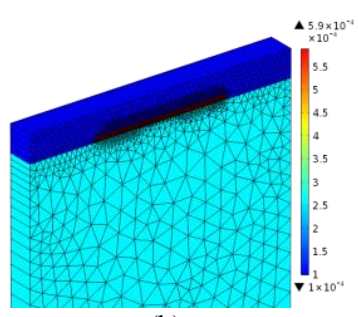

(b)

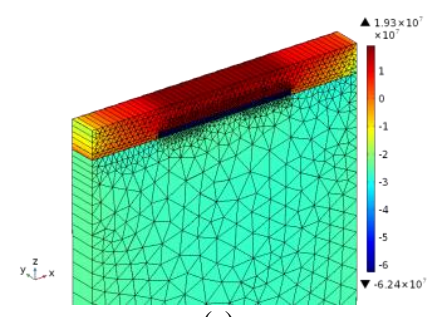

(c)

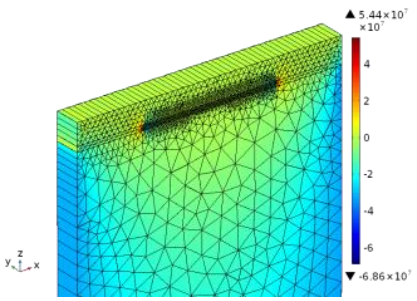

(e)

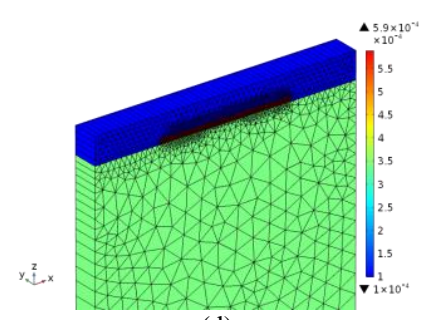

(d)

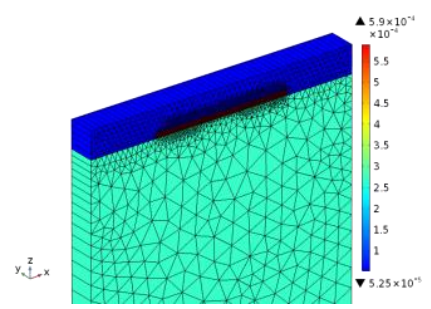

(f)
Fig. 3. The thermal stress and strain distributions in the $x$ direction ( $a$ and $b$ ). The thermal stress and strain distributions in the y direction (c and d). The thermal stress and strain distributions in the $\mathrm{z}$ direction (e and $\mathrm{f}$ ).

The effective material constants under a thermal biasing field are defined by:

$$
\begin{aligned}
& G_{L \gamma M \alpha}^{\delta}=c_{L \gamma M \alpha}^{0}+c_{L \gamma M \alpha}^{1} \Delta \Theta+c_{L \gamma M \alpha A B}^{0} S_{A B}^{\delta}+c_{L K M \alpha}^{0} w_{\gamma, K}^{\delta}+c_{L \gamma K M}^{0} w_{\alpha, K}^{\delta} \\
& R_{i j}^{\delta}=e_{i j}^{0}+e_{i j}^{1} \Delta \Theta+e_{i j k}^{0} S_{k}^{\delta}+e_{i j}^{0} w^{\delta} \\
& N_{i j}^{\delta}=\varepsilon_{i j}^{0}+\varepsilon_{i j}^{1} \Delta \Theta+\varepsilon_{i j k}^{0} E_{k}^{\delta}
\end{aligned}
$$

where $\Delta \Theta=\Theta-\Theta_{0}, c_{L \gamma M \alpha}^{0}, e_{i j}^{0}, \varepsilon_{i j}^{0}, c_{L>M \alpha A B}^{0}, e_{i j k}^{0}$, and $\varepsilon_{i j k}^{0}$ are the fundamental elastic constants, piezoelectric constants, dielectric constants, the third-order elastic, the third-order piezoelectric, and dielectric constants, respectively. $c_{L \gamma M \alpha}^{1}, \boldsymbol{e}_{i j}^{1}$, and $\varepsilon_{i j}^{1}$ are the first-order temperature derivatives of the fundamental elastic constants, piezoelectric constants, and dielectric constants, respectively. $S_{k}^{\delta}, w^{\delta}$, and $E_{k}^{\delta}$ are the thermal strain, displacement gradient, and initial electrical field of each mesh node in the calculation domain, which are multiplied by the third-order constants and fundamental constants as nonlinear dynamic increments caused by the thermal field. Therefore, each node has its own effective material constants. It is noteworthy that the thermal stress and strain tensors of each mesh node in the substrate, wave-guiding layer and electrodes are calculated using the stationary analysis at a given temperature $\Theta$.

The effective elastic and piezoelectric constants have lower symmetry than fundamental constants due to the third-order nonlinear components. Therefore, the effective elastic constant is a $9 \times 9$ matrix $G_{9 \times 9}$, and the effective piezoelectric constant is a $3 \times 9$ matrix $R_{3 \times 9}$ [18]. However, the general dimensions of the elastic matrix and piezoelectric matrix in the FEM piezoelectric coupling module are $6 \times 6$ and $3 \times 6$, which do not meet the requirements for the effective elastic matrix and effective piezoelectric matrix. In this model, the dimensions of the effective elastic and piezoelectric matrixes with nonlinear terms are defined flexibly using weak form expressions. The effective elastic matrix, the effective piezoelectric matrix, and 
the rules employed to compress indexes for the effective elastic matrix are elucidated in the appendix.

The thermal expansion effect with geometric nonlinearity is added into the model based on the coupling of the thermal field and solid mechanical field. The interfaces among the substrate, layer, and electrodes are defined as follows:

$$
\begin{gathered}
\Gamma_{q A l}=\Omega_{q} \cap \Omega_{A l} \\
\Gamma_{q l}=\Omega_{q} \cap \Omega_{\text {layer }} \\
\Gamma_{l A l}=\Omega_{\text {layer }} \cap \Omega_{\text {lAl }}
\end{gathered}
$$

The stress-free boundary condition is applied at the top surface of the wave-guiding layer. Continuous boundary conditions are applied to the displacements and stresses at the interfaces of the substrate, electrode, and wave-guiding layer as follows:

$$
\begin{aligned}
& T_{\Gamma_{q A l}}(z-)=T_{\Gamma_{q A l}}(z+) \quad u_{\Gamma_{q A l}}(z-)=u_{\Gamma_{q A l}}(z+) \\
& T_{\Gamma_{q l}}(z-)=T_{\Gamma_{q l}}(z+) \quad u_{\Gamma_{q l}}(z-)=u_{\Gamma_{q l}}(z+) \\
& T_{\Gamma_{\mid A I}}(z-)=T_{\Gamma_{\mid A I}}(z+) \quad u_{\Gamma_{\mid A l}}(z-)=u_{\Gamma_{|A|}}(z+) \\
& T_{\Gamma_{l A l}}(x-)=T_{\Gamma_{L A l}}(x+) \quad u_{\Gamma_{L A l}}(x-)=u_{\Gamma_{\mid A l}}(x+)
\end{aligned}
$$

The wave propagation is solved based on the simultaneous solution of the nonlinear piezoelectric constitutive equations and the motion equation. The weak form equation is integrated by parts to convert a body integration to a surface integration over a solution domain $\Omega$. The interface boundary conditions are imported into the weak form equations as follows:

$$
\left\{\begin{array}{l}
\iiint_{\Omega_{q}} \rho \omega^{2} u v \mathrm{~d} \Omega=\iint_{\Gamma_{q A l}} T_{\Gamma} v \mathrm{~d} \Gamma+\iint_{\Gamma_{q l}} T_{\Gamma} v \mathrm{~d} \Gamma-\iiint_{\Omega_{q}}\left(\nabla \bar{T}^{\delta}+G_{i j}^{\delta} \nabla u_{j}^{\delta}+R_{i k}^{\delta} \nabla \varphi_{k}^{\delta}\right) v \mathrm{~d} \Omega \\
\iiint_{\Omega_{l a g e r}} \rho \omega^{2} u v \mathrm{~d} \Omega=\iint_{\Gamma_{L A l}} T_{\Gamma} v \mathrm{~d} \Gamma+\iint_{\Gamma_{q l}} T_{\Gamma} v \mathrm{~d} \Gamma-\iiint_{\Omega_{l q p e r}}\left(\nabla \bar{T}^{\delta}+G_{i j}^{\delta} \nabla u_{j}^{\delta}+R_{i k}^{\delta} \nabla \varphi_{k}^{\delta}\right) v \mathrm{~d} \Omega(11) \\
\iiint_{\Omega_{A l}} \rho \omega^{2} u v \mathrm{~d} \Omega=\iint_{\Gamma_{q A l}} T_{\Gamma} v \mathrm{~d} \Gamma+\iint_{\Gamma_{l a l}} T_{\Gamma} v \mathrm{~d} \Gamma-\iiint_{\Omega_{A l}}\left(\nabla \bar{T}^{\delta}+G_{i j}^{\delta} \nabla u_{j}^{\delta}\right) v \mathrm{~d} \Omega
\end{array}\right.
$$

where $\rho$ and $\omega$ are the density and angular frequency. $T_{\Gamma}$ is the surface stress, which is continuous across the interface of different materials. $\Gamma$ is the surface area of the domain $\Omega$. Therefore, boundary conditions of the substrate, electrode, and layer are specified in the weak form equations. In the same way, the weak form electrical boundary conditions are imported as follows:

$$
\left\{\begin{array}{l}
0=-\iint_{\Gamma_{q A l}} \sigma_{\Gamma} v \mathrm{~d} \Gamma-\iint_{\Gamma_{q l}} \sigma_{\Gamma} v \mathrm{~d} \Gamma+\iiint_{\Omega_{q}}\left(R_{i j}^{\delta} \nabla u_{j}^{\delta}-N_{i k}^{\delta} \nabla \varphi_{k}^{\delta}\right) v \mathrm{~d} \Omega \\
0=-\iint_{\Gamma_{l A l}} \sigma_{\Gamma} v \mathrm{~d} \Gamma-\iint_{\Gamma_{q l}} \sigma_{\Gamma} v \mathrm{~d} \Gamma+\iiint_{\Omega_{\text {layer }}}\left(R_{i j}^{\delta} \nabla u_{j}^{\delta}-N_{i k}^{\delta} \nabla \varphi_{k}^{\delta}\right) v \mathrm{~d} \Omega \\
0=-\iint_{\Gamma_{q A l}} \sigma_{\Gamma} v \mathrm{~d} \Gamma-\iint_{\Gamma_{l A l}} \sigma_{\Gamma} v \mathrm{~d} \Gamma+\iiint_{\Omega_{A l}} N_{i k}^{\delta} \nabla \varphi_{k}^{\delta} v \mathrm{~d} \Omega
\end{array}\right.
$$

where $\sigma_{\Gamma}$ is the surface electric charge at the interfaces. Descriptions of the key modeling steps are given as follows:

1. Defining the material properties, including the effective elastic constants $(9 \times 9$ matrix), effective piezoelectric constants ( $3 \times 9$ matrix) and effective dielectric constants, which are functions of the temperature. The environmental temperature and thermal expansion coefficients are also set.

2. Defining the weak form PDEs for piezoelectric and elastic materials, including the stress and strain tensors $(1 \times 9$ vector $)$ in Lagrange coordinate.

3. Updating the thermal stress and strain tensors resulting from the stationary analysis at the given temperature.

4. Using the modal analysis, the relative frequency shift caused by temperature is calculated.

\section{EXPERIMENTAL RESULTS AND DISCUSSION}

In the following part, $25^{\circ} \mathrm{C}$ is set as the reference temperature. The fundamental material constants, the thirdorder material constants and temperature coefficients of quartz, $\mathrm{ZnO}, \mathrm{SiO}_{2}$ and aluminum are taken from the references [19]-[25].

\section{A. $\mathrm{SiO}_{2} / 35.2^{\circ} Y X+90^{\circ}$-cut quartz}

The frequency-temperature characteristic of the Love wave mode at the resonance frequency on $\mathrm{SiO}_{2} / 35.2^{\circ} \mathrm{YX}+90^{\circ}$-cut quartz structure is shown in Fig. 4. $\mathrm{SiO}_{2}$ works as a waveguiding layer. The wavelength is $48.4 \mu \mathrm{m}$. The thickness of the aluminum electrode is $150 \mathrm{~nm}$, and the metallization ratio is $0.5 . \mathrm{SiO}_{2}$ thickness is $1 \mu \mathrm{m}$. The experimental data are taken from [9]. In this section, the FEM Campbell model, referred to the FEM model using the temperature coefficients of quartz according to the Campbell method [10], [26], is also calculated for comparison. This model considers the variation of material constants with temperature and the impact of the thermal expansion on the propagation of surface acoustic waves. It is seen that the simulation results of the weak form nonlinear model are more consistent with the experimental data than that of the FEM Campbell model. The first-order TCF of Love wave mode is $0.47 \mathrm{ppm} /{ }^{\circ} \mathrm{C}$, and the turnover temperature is $0{ }^{\circ} \mathrm{C}$. Although the relative frequency shift is less than $100 \mathrm{ppm}$ in a temperature range from $-40{ }^{\circ} \mathrm{C}$ to $40{ }^{\circ} \mathrm{C}$, the frequency shift increases rapidly over the room temperature. When the temperature increases from $50{ }^{\circ} \mathrm{C}$ to $100{ }^{\circ} \mathrm{C}$, the relative frequency shift decreases from $-100 \mathrm{ppm}$ to $-600 \mathrm{ppm}$. When the $\mathrm{SiO}_{2}$ thickness is $3.158 \mu \mathrm{m}$, the frequency-temperature dependence of the resonance frequency is shown in Fig. 5. The turnover temperature rises to $60{ }^{\circ} \mathrm{C}$. The computed turnover temperature of FEM Campbell model is $20{ }^{\circ} \mathrm{C}$ below the experimental turnover temperature. The theoretical data of the weak form model match well with the experimental results. However, the electromechanical coupling coefficient $K^{2}$ is only $0.07 \%$ due to the nonpiezoelectricity of the $\mathrm{SiO}_{2}$ layer. 


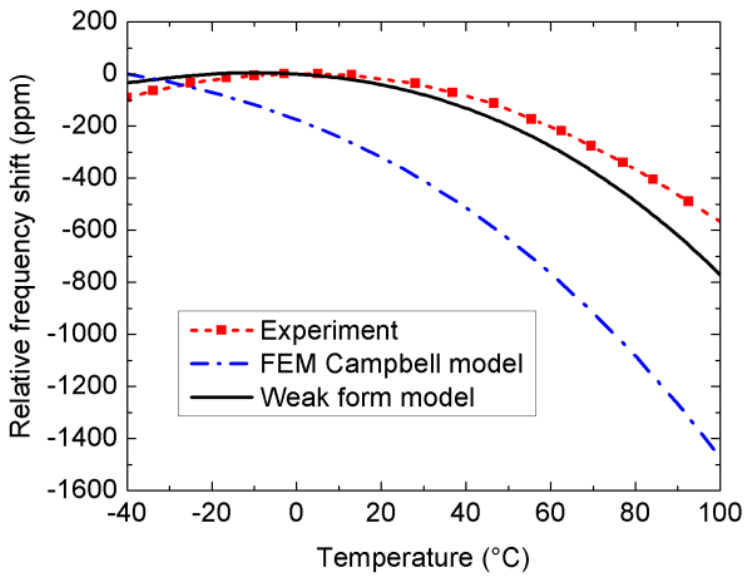

Fig. 4. Comparison of theoretical and experimental frequency-temperature characteristics of Love wave on $\mathrm{SiO}_{2} / 35.2^{\circ} \mathrm{YX}+90^{\circ}$-cut quartz. The $\mathrm{SiO}_{2}$ thickness is $1 \mu \mathrm{m}$.

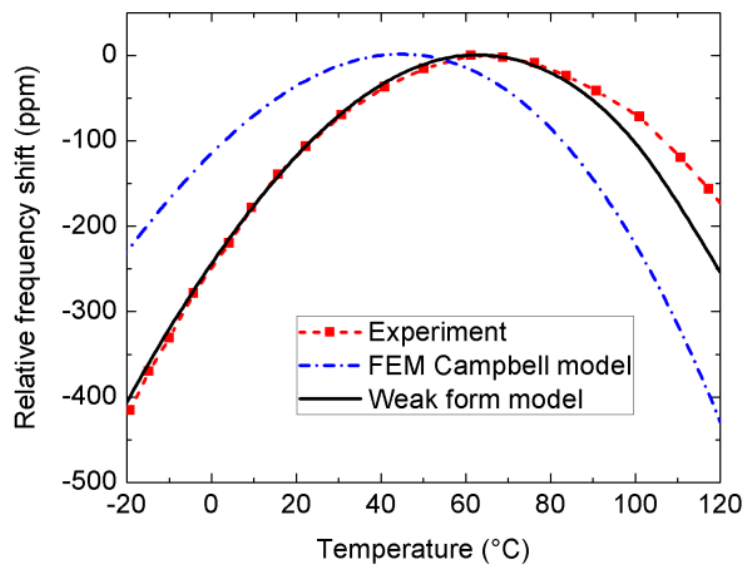

Fig. 5. Comparison of theoretical and experimental frequency-temperature characteristics of Love wave on $\mathrm{SiO}_{2} / 35.2^{\circ} \mathrm{YX}+90^{\circ}$-cut quartz. $\mathrm{SiO}_{2}$ thickness is $3.158 \mu \mathrm{m}$.

\section{B. $\mathrm{ZnO} / \mathrm{ST}+90^{\circ}$-cut quartz}

Compared to the $\mathrm{SiO}_{2}$ wave-guiding layer, a $\mathrm{ZnO}$ thin film has the advantages of piezoelectric property and lower acoustic velocity. The velocity of shear wave in the $\mathrm{ZnO}$ thin film is $2578 \mathrm{~m} / \mathrm{s}$, slower than the quartz substrate's. Thus, a $\mathrm{ZnO}$ film is employed as a wave-guiding layer to confine the Love wave energy near the surface.

The shear-horizontal $(\mathrm{SH})$ wave mode at the resonance frequency on $\mathrm{ST}+90^{\circ}$-cut quartz has a positive first-order TCF around $+30 \mathrm{ppm} /{ }^{\circ} \mathrm{C}$, as shown in Fig. 6 . The results of weak form model are consistent with the experimental data. Fig. 7 depicts the measured $\mathrm{S}_{11}$ of the $\mathrm{Al} / \mathrm{ST}+90^{\circ}$-cut quartz resonator. It can be seen that the resonance frequency is $483.48 \mathrm{MHz}$ at the room temperature. There is an obvious resonance frequency shift when the temperature increases from $25{ }^{\circ} \mathrm{C}$ to $60{ }^{\circ} \mathrm{C}$. The resonance frequency increases to $483.99 \mathrm{MHz}$ at $60{ }^{\circ} \mathrm{C}$, confirming the positive TCF value of $\mathrm{ST}+90^{\circ}$-cut quartz. Thus, to achieve a zero TCF structure, the negative TCF of the $\mathrm{ZnO}$ layer is compensated by the positive TCF value of the $\mathrm{ST}+90^{\circ}$-cut quartz. Fig. 8 illustrates the electromechanical coupling coefficient $K^{2}$ and the first-order
TCF for the Love wave mode as a function of the normalized $\mathrm{ZnO}$ film thickness on the $\mathrm{ZnO} / \mathrm{ST}+90^{\circ}$-cut quartz structure. The wavelength $(\lambda)$ is $10 \mu \mathrm{m}$. It is found that $K^{2}$ increases to the maximum value of $0.29 \%$ at $\mathrm{h}_{\mathrm{ZnO}}=0.03 \lambda$ and then decreases. The first-order TCF decreases with the growth of $\mathrm{ZnO}$ thickness due to the negative temperature coefficients of $\mathrm{ZnO}$ elastic constants. The zero TCF is obtained when $\mathrm{ZnO}$ thickness is $0.05 \lambda$, and $K^{2}$ is $0.25 \%$. The phase velocity decreases from $4870 \mathrm{~m} / \mathrm{s}$ to $3660 \mathrm{~m} / \mathrm{s}$ with the increase of $\mathrm{ZnO}$ thickness from $0.02 \lambda$ to $0.1 \lambda$.

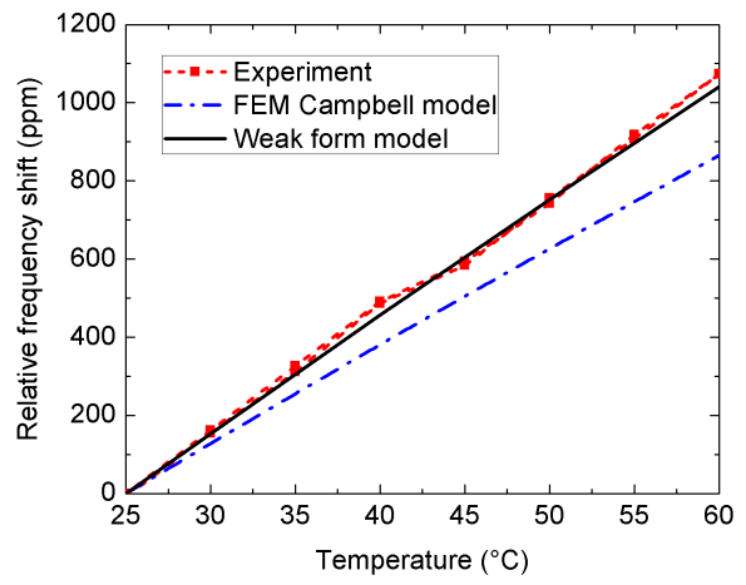

Fig. 6. Theoretical and experimental frequency-temperature characteristics of SH wave on $\mathrm{ST}+90^{\circ}$-cut quartz. The $\mathrm{Al}$ electrode thickness is $100 \mathrm{~nm}$.

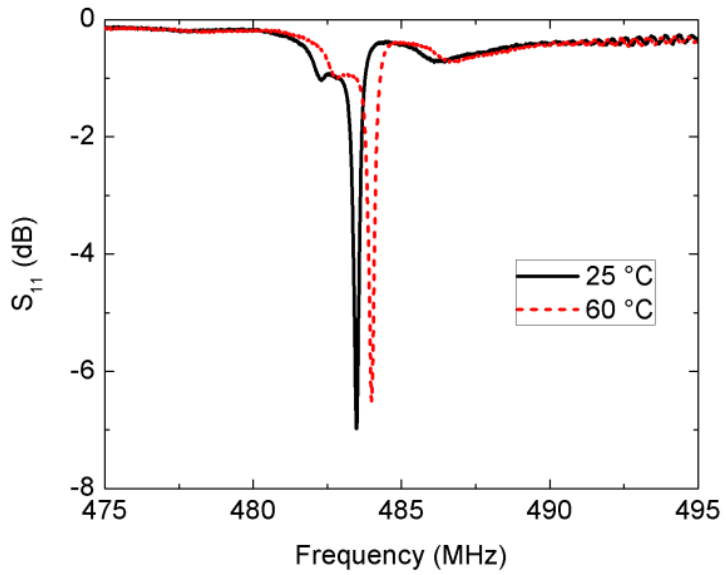

Fig. 7. The measured $\mathrm{S}_{11}$ of the $\mathrm{Al}$ electrodes $/ \mathrm{ST}+90^{\circ}$-cut quartz resonator.

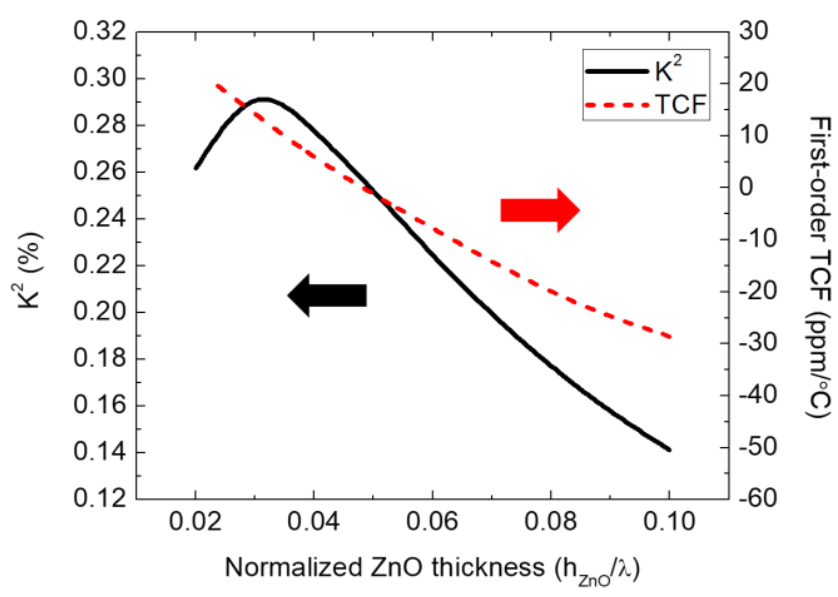


Fig. 8. The electromechanical coupling coefficient and phase velocity of Love wave mode on $\mathrm{ZnO} / \mathrm{ST} 90^{\circ}$-cut quartz as a function of the normalized $\mathrm{ZnO}$ thickness, $\mathrm{h}_{\mathrm{Al}}=0.01 \lambda, \mathrm{r}=0.5$.

When the $\mathrm{ZnO}$ thickness is $0.05 \lambda$, the thermal strains of the interfaces in $\mathrm{ZnO} / \mathrm{ST}+90^{\circ}$-cut quartz structure are illustrated in Fig. 9. The symbol $x$ is the propagation direction, and $y$ is the aperture direction. The thermal strain at the interface of layers is determined by the thermal expansions of surrounding materials. At the reference temperature of $25^{\circ} \mathrm{C}$, the thermal strains of all nodes are zero. Due to the anisotropy of quartz, the thermal strains in the $\mathrm{x}, \mathrm{y}, \mathrm{z}$ directions are different. The thermal strains of the interfaces increase with the temperature increasing or decreasing far away from $25^{\circ} \mathrm{C}$, which affects the nonlinear components in the effective constants. In addition, the thermal strains of the interface $\Gamma_{\mathrm{qAl}}$ are all larger than those of interface $\Gamma_{\mathrm{lAl}}$ and interface $\Gamma_{\mathrm{ql}}$, because the thermal expansion coefficients of the quartz $\left(\alpha_{11}=13.71 \times 10^{-}\right.$ $\left.{ }^{6} /{ }^{\circ} \mathrm{C}, \alpha_{33}=7.48 \times 10^{-6} /{ }^{\circ} \mathrm{C}\right)$ and the $\mathrm{Al}$ electrode $\left(\alpha_{11}=18 \times 10^{-6} /{ }^{\circ} \mathrm{C}\right)$ are larger than that of $\mathrm{ZnO}$ thin film $\left(\alpha_{11}=4 \times 10^{-6} /{ }^{\circ} \mathrm{C}\right.$, $\left.\alpha_{33}=2.1 \times 10^{-6} /{ }^{\circ} \mathrm{C}\right) \quad[19], \quad[20]$. The thermal expansion coefficients of quartz, $\mathrm{Al}$, and $\mathrm{ZnO}$ are on the same order of magnitude, and none of them are negligible. The thermal strain of the interface $\Gamma_{\mathrm{qAl}}$ in y direction is the maximum at a fixed temperature.

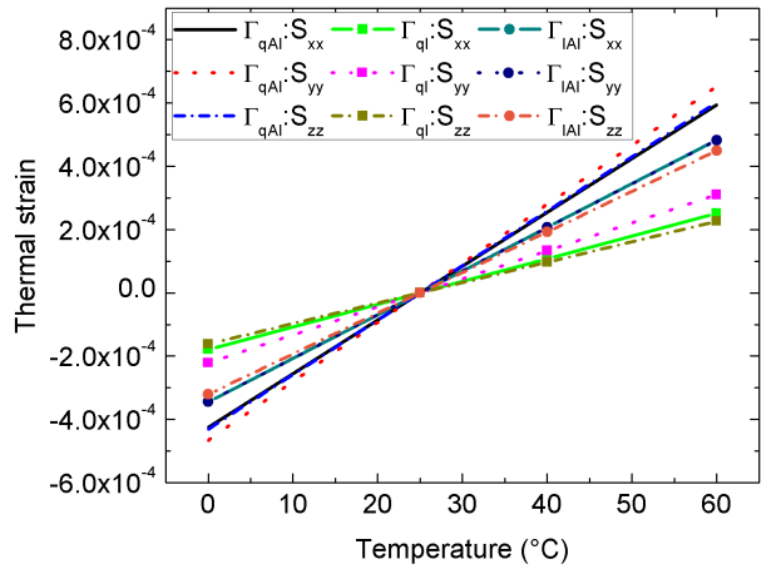

Fig. 9. Thermal strains of interfaces versus the temperature on $\mathrm{ZnO} / \mathrm{ST}+90^{\circ}$ cut quartz. $\mathrm{h}_{\mathrm{ZnO}}=0.05 \lambda, \mathrm{h}_{\mathrm{Al}}=0.01 \lambda, \mathrm{r}=0.5$.

The average thermal stresses of the interfaces are illustrated in Fig. 10. At the reference temperature $25^{\circ} \mathrm{C}$, the thermal stresses are zero. They increase with the temperature changes far away from $25{ }^{\circ} \mathrm{C}$. It is found that the thermal stresses mainly concentrate on the surface of the metal electrode, especially on the interface between the electrode and quartz in the aperture direction. The thermal stress in the aperture direction of an interface is larger than those in other directions. Moreover, the thermal stress on the interface $\Gamma_{\mathrm{qAl}}$ between the quartz and electrode in y direction is twice that on the interface $\Gamma_{\text {lAl }}$ between the $\mathrm{ZnO}$ layer and electrode in the aperture direction.

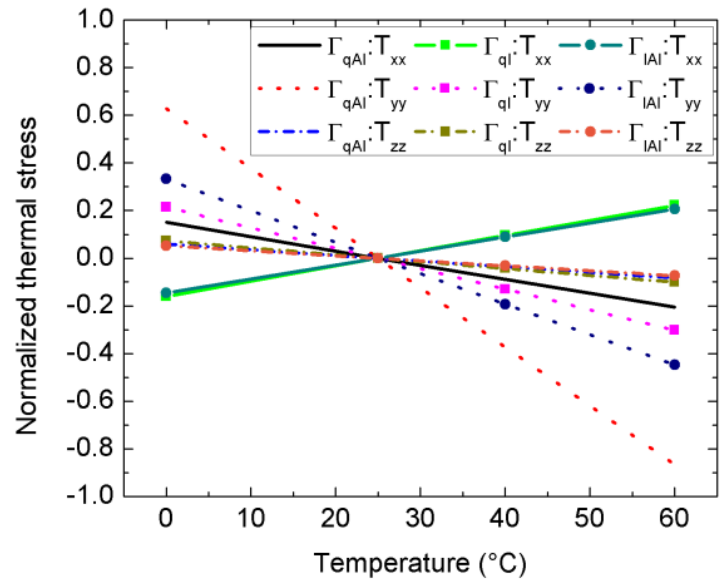

Fig. 10. Thermal stress of interfaces on $\mathrm{ZnO} / \mathrm{ST}+90^{\circ}$-cut quartz. $\mathrm{h}_{\mathrm{ZnO}}=0.05 \lambda$, $\mathrm{h}_{\mathrm{Al}}=0.01 \lambda, \mathrm{r}=0.5$.

Based on the simulation results of the model, one-port Love wave mode resonators on the $\mathrm{ZnO} / \mathrm{ST}+90^{\circ}$-cut quartz structure have been fabricated. Realizations are done using conventional ultraviolet (UV) lithography and lift-off technique. The wavelength of the fabricated device is $10 \mu \mathrm{m}$. The thickness of the aluminum electrode is $100 \mathrm{~nm}$, and the metallization ratio is 0.5 . The number of IDT pairs is 100 , and the number of reflective gratings is 200 on both sides of the IDTs. The aperture length of the resonator is $566 \mu \mathrm{m}$. Subsequently, a $\mathrm{ZnO}$ layer of $500 \mathrm{~nm}$ was deposited, and patterned by a maskless lithography process and lift-off. The $\mathrm{ZnO}$ film was obtained by radio-frequency (RF) sputtering of a 4 inch oxygen rich target at $150 \mathrm{~W}$ with $3 \times 10^{-3}$ mbar and 8 $\mathrm{cm}^{3} / \mathrm{min}$ flow rate of both argon (Ar) and oxygen $\left(\mathrm{O}_{2}\right)$.

As shown in Fig. 11, the calculated thermal sensitivity of the Love wave model at the resonance frequency is consistent with the experimental result. The solid line represents the simulation data, and the dotted lines indicates the increasing and decreasing temperatures for the two experimental curves. The arrows indicate the temperature up and down. A stable frequency-temperature characteristic is obtained with the firstorder TCF $-1.26 \mathrm{ppm} /{ }^{\circ} \mathrm{C}$. The relative frequency shift is less than $50 \mathrm{ppm}$ in a temperature range from $25^{\circ} \mathrm{C}$ to $60^{\circ} \mathrm{C}$. The measured $\mathrm{S}_{11}$ parameters of the Love wave device at the room temperature and at $60{ }^{\circ} \mathrm{C}$ are shown in Fig. 12. The resonance frequency is $409.68 \mathrm{MHz}$ at the room temperature. It can be seen that there is little shift of the resonance frequency when the temperature increases from $25^{\circ} \mathrm{C}$ to $60{ }^{\circ} \mathrm{C}$, confirming the near zero TCF of the achieved structure. 


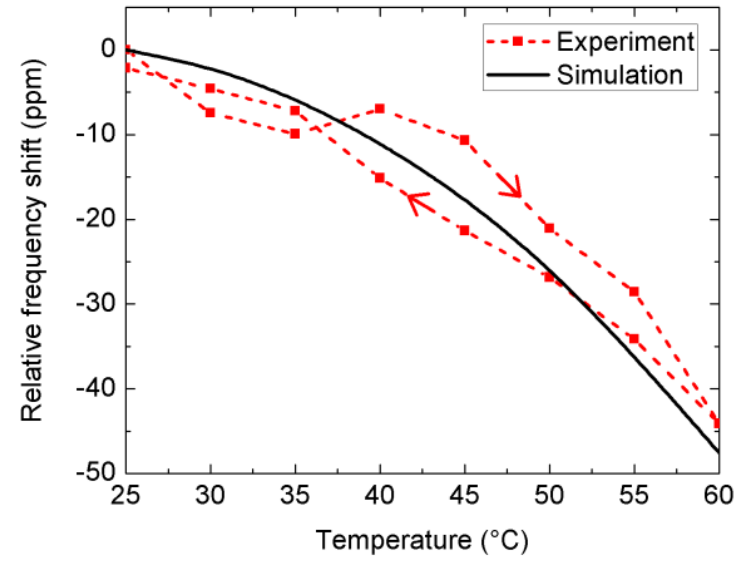

Fig. 11. The simulation and experimental frequency temperature curves of Love wave mode on $\mathrm{ZnO} / \mathrm{ST}+90^{\circ}$-cut quartz with $\mathrm{ZnO}$ thickness $500 \mathrm{~nm}$.

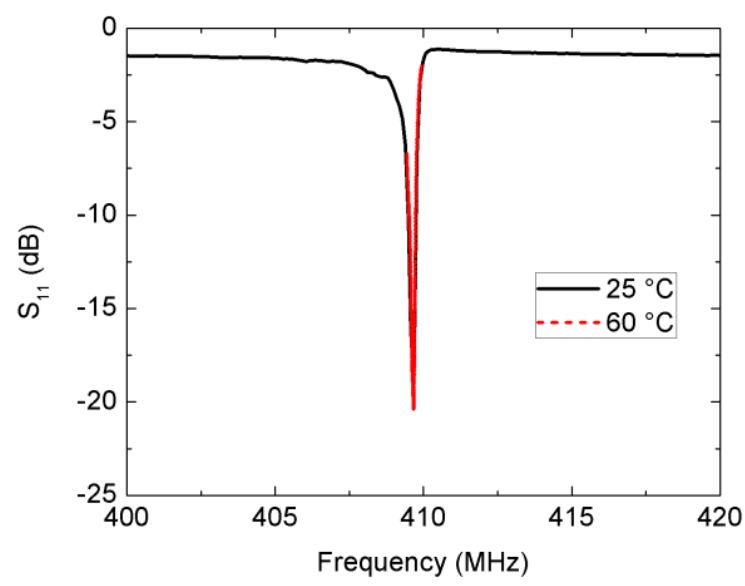

Fig. 12. The measured $\mathrm{S}_{11}$ of the $\mathrm{ZnO} / \mathrm{ST}+90^{\circ}$-cut quartz resonator.

A multi-layered structure of $\mathrm{ZnO} /\left(\mathrm{IDT}+\mathrm{SiO}_{2}\right) /-$ $52^{\circ} \mathrm{YX}+90^{\circ}$-cut quartz with embedded electrodes is considered for temperature compensation to design a Love wave device with a wider range of thermal stability. Considering that the $\mathrm{SH}$ wave resonator on $-52^{\circ} \mathrm{YX}+90^{\circ}$-cut quartz reduces the frequency temperature fluctuation to a half of the ST-cut quartz [27], this cut provides the possibility of achieving a better temperature stability for Love wave devices. Amorphous $\mathrm{SiO}_{2}$ is filled between the electrodes, and the upper surface of $\mathrm{SiO}_{2}$ is polished to keep flat. The $\mathrm{ZnO}$ is deposited on the polished flat $\mathrm{SiO}_{2}$ layer. Temperature compensation is obtained with an optimized combination of $\mathrm{ZnO}$ and $\mathrm{SiO}_{2}$ layers. The wavelength $(\lambda)$ is $10 \mu \mathrm{m}$. $\mathrm{ZnO}$ thicknesses is $0.024 \lambda . \mathrm{SiO}_{2}$ and aluminum electrode thicknesses are both $0.03 \lambda$. The metallization ratio is 0.5 . The calculated frequency temperature curve of the resonance frequency is shown in Fig. 13. The relative frequency shift is less than $20 \mathrm{ppm}$ over a wide temperature range of $-40{ }^{\circ} \mathrm{C}-60{ }^{\circ} \mathrm{C}$. Its temperature stability is superior to that of the $\mathrm{ZnO} / \mathrm{ST}+90^{\circ}$-cut quartz. The enhanced of thermal stability could be explained by the positive temperature coefficients of added $\mathrm{SiO}_{2}$ layer. However, the reflective coefficient of this structure is 0.00051 . It is smaller than that of $\mathrm{ZnO} / \mathrm{ST}+90^{\circ}$-cut quartz $(0.055)$.

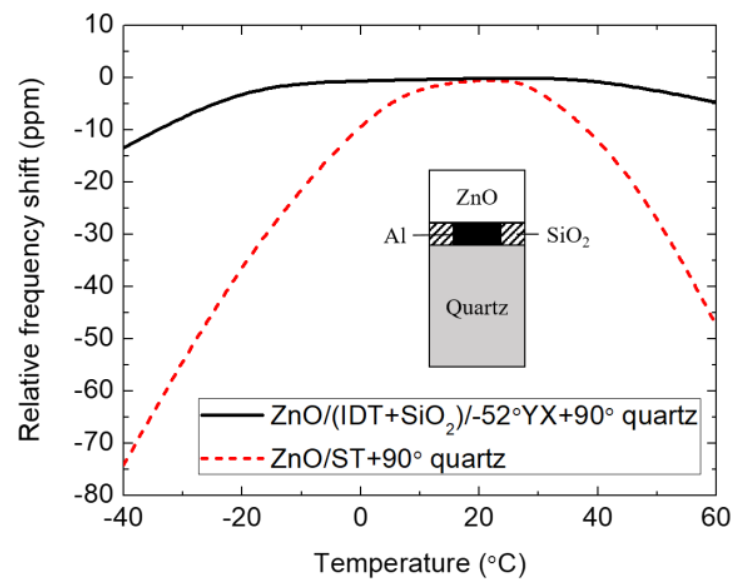

Fig. 13. The frequency temperature curve on $\mathrm{ZnO} /\left(\mathrm{IDT}+\mathrm{SiO}_{2}\right) /-52^{\circ} \mathrm{YX}+90^{\circ}-$ cut quartz.

\section{CONCLUSION}

The weak form nonlinear model is developed to accurately compute the thermal sensitivity of the Love wave mode on the quartz substrates. The third-order material constants, thermal strains, and stresses are taken into account with the coupling of thermal and mechanical fields. The temperature behavior of the Love wave device is calculated. The effect of $\mathrm{ZnO}$ thickness on the frequency-temperature characteristics of Love wave mode is studied for temperature compensation. The analytical results of weak form model are in good agreement with the experimental data. The results contribute to designing the Love wave resonators as wireless sensors with a wide and stable operating temperature range.

\section{APPENDIX}

Table I lists the rule used to compress indexes for the effective elastic matrix.

TABLE I. INDEX ABBREVIATION RULE [18]

\begin{tabular}{|c|c|c|}
\hline $11 \rightarrow 1$ & $22 \rightarrow 2$ & $33 \rightarrow 3$ \\
\hline $23 \rightarrow 4$ & $31 \rightarrow 5$ & $12 \rightarrow 6$ \\
\hline $32 \rightarrow 7$ & $13 \rightarrow 8$ & $21 \rightarrow 9$ \\
\hline
\end{tabular}

The rotated $\mathrm{YX}+90^{\circ}$-cut quartz is adopted as the substrate in this model. The effective elastic matrix of rotated $\mathrm{YX}+90^{\circ}$ cut quartz is defined as follows:

$$
G=\left[\begin{array}{ccccccccc}
G_{11} & G_{12} & G_{13} & 0 & G_{15} & 0 & 0 & G_{18} & 0 \\
G_{12} & G_{11} & G_{13} & 0 & G_{25} & 0 & 0 & G_{28} & 0 \\
G_{13} & G_{13} & G_{33} & 0 & G_{35} & 0 & 0 & G_{38} & 0 \\
0 & 0 & 0 & G_{44} & 0 & G_{46} & G_{47} & 0 & G_{49} \\
G_{15} & G_{25} & G_{35} & 0 & G_{55} & 0 & 0 & G_{58} & 0 \\
0 & 0 & 0 & G_{46} & 0 & G_{66} & G_{67} & 0 & G_{69} \\
0 & 0 & 0 & G_{47} & 0 & G_{67} & G_{77} & 0 & G_{79} \\
G_{18} & G_{28} & G_{38} & 0 & G_{58} & 0 & 0 & G_{88} & 0 \\
0 & 0 & 0 & G_{49} & 0 & G_{69} & G_{79} & 0 & G_{99}
\end{array}\right]
$$


The effective piezoelectric matrix of rotated $\mathrm{YX}+90^{\circ}$-cut quartz is defined as follows:

$$
R=\left[\begin{array}{lllllllll}
0 & 0 & 0 & R_{14} & 0 & R_{16} & R_{17} & 0 & R_{19} \\
R_{21} & R_{22} & R_{23} & 0 & R_{25} & 0 & 0 & R_{28} & 0 \\
0 & 0 & 0 & R_{34} & 0 & R_{36} & R_{37} & 0 & R_{39}
\end{array}\right]
$$

The effective elastic matrix of $\mathrm{ZnO}$ wave-guiding layer is defined as follows:

$$
\left[\begin{array}{ccccccccc}
G_{11} & G_{12} & G_{13} & 0 & 0 & 0 & 0 & 0 & 0 \\
G_{12} & G_{11} & G_{13} & 0 & 0 & 0 & 0 & 0 & 0 \\
G_{13} & G_{13} & G_{33} & 0 & 0 & 0 & 0 & 0 & 0 \\
0 & 0 & 0 & G_{44} & 0 & 0 & G_{47} & 0 & 0 \\
0 & 0 & 0 & 0 & G_{44} & 0 & 0 & G_{58} & 0 \\
0 & 0 & 0 & 0 & 0 & G_{66} & 0 & 0 & G_{69} \\
0 & 0 & 0 & G_{47} & 0 & 0 & G_{44} & 0 & 0 \\
0 & 0 & 0 & 0 & G_{58} & 0 & 0 & G_{44} & 0 \\
0 & 0 & 0 & 0 & 0 & G_{69} & 0 & 0 & G_{66}
\end{array}\right]
$$

The effective elastic matrix of aluminum electrode is defined as follows:

$$
\left[\begin{array}{ccccccccc}
G_{11} & G_{12} & G_{12} & 0 & 0 & 0 & 0 & 0 & 0 \\
G_{12} & G_{11} & G_{12} & 0 & 0 & 0 & 0 & 0 & 0 \\
G_{12} & G_{12} & G_{11} & 0 & 0 & 0 & 0 & 0 & 0 \\
0 & 0 & 0 & G_{66} & 0 & 0 & G_{66} & 0 & 0 \\
0 & 0 & 0 & 0 & G_{66} & 0 & 0 & G_{66} & 0 \\
0 & 0 & 0 & 0 & 0 & G_{66} & 0 & 0 & G_{66} \\
0 & 0 & 0 & G_{66} & 0 & 0 & G_{66} & 0 & 0 \\
0 & 0 & 0 & 0 & G_{66} & 0 & 0 & G_{66} & 0 \\
0 & 0 & 0 & 0 & 0 & G_{66} & 0 & 0 & G_{66}
\end{array}\right]
$$

\section{REFERENCES}

[1] M. I. Rocha-Gaso, C. March-Iborra, Á. Montoya-Baides, and A. ArnauVives, "Surface generated acoustic wave biosensors for the detection of pathogens: A review," Sensors, vol. 9, no. 7, pp. 5740-5769, July. 2009.

[2] A. Kittmann, P. Durdaut, S. Zabel, J. Reermann, J. Schmalz, B. Spetzler, D. Meyners, N. X. Sun, J. McCord, M. Gerken, G. Schmidt, M. Höft, R. Knöchel, F. Faupel, and E. Quandt, "Wide band low noise Love wave magnetic field sensor system." Scientific reports, vol. 8, no. 1, pp. 278-285, Jan. 2018.

[3] Y. Yang, Q. Z. Zhang, Y. R. Shi, T. Han, P. Li, and Y. M. Wen, "Love Wave Mode Resonator with a Wide Stable Temperature Range," in Proc. IEEE Ultrason. Symp., Sep. 2018, pp. 1-4, Doi: 10.1109/ULTSYM.2018.8580142.

[4] F. Herrmann, M. Weihnacht, S. Buttgenbach, "Properties of sensors based on shear-horizontal surface acoustic waves in $\mathrm{LiTaO}_{3} / \mathrm{SiO}_{2} /$ and quartz/ $\mathrm{SiO}_{2}$ /structures," IEEE Trans. Ultrason., Ferroelectr., Freq. Control, vol. 48, no. 1, pp. 268-273, Sep. 2001.

[5] I. D. Avramov, K. D. Esmeryan, "Thermal sensitivity of solid polymer coated surface transverse/Love wave based resonators on AT-cut quartz for sensor applications." Sensors \& Transducers, vol. 147, no. 12, pp. 15-26, Dec. 2012.

[6] K. K. Zadeh, A. Trinchi, W. Wlodarski, and A. Hollanda, "A novel Love-mode device based on a $\mathrm{ZnO} / \mathrm{ST}$-cut quartz crystal structure for sensing applications," Sensor. Actuat. A-Phys., vol. 100, no.2-3, pp. 135-143, Sep. 2002.

[7] B. Jakoby, J. Bastemeijer, M. J. Vellekoop, "Temperature-compensated Love-wave sensors on quartz substrates," Sensors and Actuators A: Physical, vol. 82, no. 1-3, pp. 83-88, May. 2000.

[8] B. Jakoby, M. J. Vellekoop, "Substrates for zero temperature coefficient Love wave sensors." IEEE Trans. Ultrason., Ferroelectr., Freq. Control, vol. 47, no. 3, pp. 701-705, May. 2000.

[9] F. Josse, "Temperature dependence of SH-wave on rotated y-cut quartz with $\mathrm{SiO}_{2}$ overlay," IEEE Trans. Sonics, Ultrason., vol. 31, no. 3, pp. 162-167, May. 1984.

[10] J. J. Campbell, W. R. Jones, "A method for estimating optimal crystal cuts and propagation directions for excitation of piezoelectric surface waves," IEEE Trans. Sonics, Ultrason., vol. 15, no. 4, pp. 209-217, Oct. 1968

[11] F. Moreira, M. E. Hakiki, F. Sarry, L. L. Brizoual, O. Elmazria, and P. Alnot, "Numerical Development of $\mathrm{ZnO} / \mathrm{Quartz}$ Love Wave Structure for Gas Contamination Detection," IEEE Sens. J., vol. 7, no. 3, pp. 336341, Feb. 2007.

[12] J. Garcia, T. Laroche, W. Daniau, E. Carry, G. Martin, S. Ballandras, and J. M. Friedt, "2-and-3D analysis of temperature effects on periodic transducers using a FEA/BEM approach," in Proc. IEEE Inter. Freq. Cont. Symp. Joint with the 22nd Euro. Freq. Time forum. IEEE, Apr. 2009, pp. 424-429, DOI: 10.1109/FREQ.2009.5168214.

[13] W. B. Wang, K. Tang, H. D. Wu, "Analysis of frequency-temperature behavior of surface acoustic wave (SAW) under periodic IDT," in Proc. the 2010 Symp. Piezo., Acoust. Wave. Dev. Appli.. IEEE, Dec. 2010, pp. 460-464, DOI: 10.1109/SPAWDA.2010.5744356.

[14] E. Gavignet, S. Ballandras, E. Bigler, "A perturbation method for modeling the thermal sensitivity of surface transverse waves," IEEE Trans. Ultrason., Ferroelectr., Freq. Control, vol. 44, no. 1, pp. 201207, Jan. 1997.

[15] B. K. Sinha, H. F. Tiersten, "First temperature derivatives of the fundamental elastic constants of quartz," J. Appl. Phys., vol. 50, no. 4, pp. 2732-2739, Apr. 1979.

[16] E. Henry-Briot, S. Ballandras, G. Marianneau, and G. Martin, "Influence of metal thickness on phase velocity and thermal sensitivity of SAW devices," IEEE Trans. Ultrason., Ferroelectr., Freq. Control, vol. 48, no. 2, pp. 538-546, Mar. 2001.

[17] B. Dulmet, R. Bourquin, "Lagrangian effective material constants for the modeling of thermal behavior of acoustic waves in piezoelectric crystals. I. Theory," J. Acoust. Soc. Am., vol. 110, no. 4, pp. 1792-1799, Oct. 2001.

[18] B. Dulmet, R. Bourquin, "Application of Lagrangian effective material constants to the study of the thermal behavior of SAW propagation in piezoelectric crystals," in Proc. IEEE Ultrason. Symp., Oct. 1994, vol. 1, pp. 331-335, DOI: 10.1109/ULTSYM.1994.401604.

[19] M. Tomar, V. Gupta, K. Sreenivas, and A. Mansingh, "Temperature stability of $\mathrm{ZnO}$ thin film SAW device on fused quartz," IEEE Trans. Dev. Mat. Re., vol. 5, no. 3, pp. 494-500, Dec. 2005.

[20] J. Bjurström, G. Wingqvist, V. Yantchev, and I. Katardjiev, "Temperature compensation of liquid FBAR sensors," J. Micromech. \& Microeng., vol. 17, no. 3, pp. 651-658, Feb. 2007.

[21] R. N. Thurston, H. J. McSkimin, P. Andreatch, "Third-order elastic coefficients of quartz," J. Appl. Phys., vol. 37, no. 1, pp. 267-275, Jan. 1966.

[22] E. Kittinger, J. Tichý, W. Friedel, "Nonlinear piezoelectricity and electrostriction of alpha quartz," J. Appl. Phys., vol. 60, no. 4, pp. 14651471, Aug. 1986.

[23] X. Wang, Y. Gu, X. Sun, H. Wang, and Y. Zhang, "Third-order elastic constants of $\mathrm{ZnO}$ and size effect in $\mathrm{ZnO}$ nanowires," J. Appl. Phys., vol. 115, no. 21, pp.115-123, Jun. 2014.

[24] E. H. Bogardus, "Third-Order Elastic Constants of $\mathrm{Ge}, \mathrm{MgO}$, and Fused $\mathrm{SiO}_{2}$," J. Appl. Phys., vol. 36, no. 8 pp. 2504-2513, Aug. 1965.

[25] S. Ballandras, E. Gavignet, E. Bigler, and E. Henry, "Temperature derivatives of the fundamental elastic constants of isotropic materials," Appl. Phys. Lett., vol. 71, no. 12, pp. 1625-1627, Sep. 1997.

[26] R. Bechmann, A. D. Ballato, and T. J. Lukaszek, "Higher-order temperature coefficients of the elastic stiffinesses and compliances of 
alpha-quartz," in Proc. IRE, Aug. 1962, vol. 50, no. 8, pp. 1812-1822, DOI: $10.1109 / J R P R O C .1962 .288222$.

[27] T. Owaki, T. Morita, "Excellent frequency stability and high-Q and small SH-type quartz SAW resonators," In Proc. IEEE Ultrason. Symp., Sep. 2005, vol. 4, pp. 1888-1891, DOI: 10.1109/ ULTSYM. 2005. 1603241.

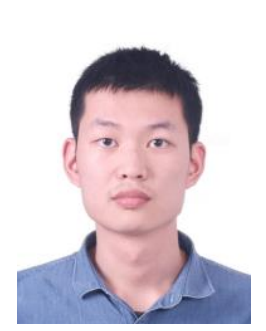

Yang Yang was born in Jiangsu, China. $\mathrm{He}$ received the $\mathrm{BE}$ degree from University of Electronic Science and Technology of China in 2015. He is currently a Ph.D. student at School of Electronic Information and Electrical Engineering in Shanghai Jiao Tong University. His current research interests include wireless surface acoustic wave sensor and signal processing.

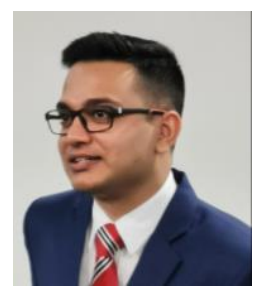

Harshad Mishra was born in Odisha, India, in 1989. He received his Ph.D. degree from Institut Jean Lamour, Université de Lorraine, Nancy, France, in 2019. Prior to that, he received his M.S. degree from IIT Madras, Chennai, India, in 2016. His research interests include the development and optimization of magnetic field sensors using micro-structured, magnetoelastic thin films and devices based on surface acoustic waves.

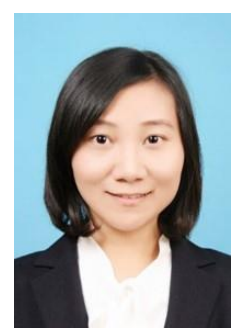

Qiaozhen Zhang was born in Anhui Province, China, in May 1988. She received her Master's degree from Huazhong University of Science \& Technology, Wuhan China, in March 2013. She enrolled in Shanghai Jiao Tong University, and joined in cooperative training project between Shanghai Jiao Tong University and Chiba University in 2015. She received $\mathrm{Ph}$. D from Shanghai Jiao Tong University in November 2018. In December 2018, she started to work as a lecturer in the College of Information, Mechanical and Electrical Engineering in Shanghai Normal University. Her current research interests include intelligent voice information processing, simulation and design of various highperformance surface acoustic wave devices, acoustic wave sensors and actuators.

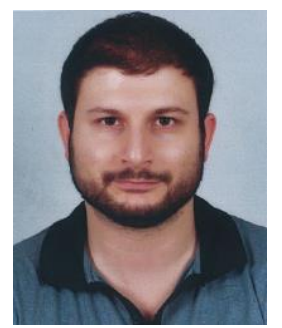

Sami Hage-Ali was born in Strasbourg, France, in 1982. He received an Engineering Degree from Ecole Centrale de Lille and a M.S in micronanotechnology from University of Lille 1 in 2005. He received another Master's Degree in international projects engineering from University of Lille 1 in
2006. He received a Ph.D. in micro-nanotechnology, acoustics and telecommunications from Ecole Centrale de Lille in 2011. In 2011, Dr Hage-Ali was awarded a Fulbright grant and became a post-doctoral fellow at the University of Illinois at Urbana-Champaign, USA. Since 2014, he is an Associate professor at Université de Lorraine and is with the Micro-nanosystems group of Institut Jean Lamour, Nancy, France. Dr Hage-Ali's areas of research are: surface acoustic wave sensors, flexible/stretchable electronics, micronanosystems, microwaves and antennas. He is currently serving as chairman of the IEEE France Section Sensors Council Chapter.

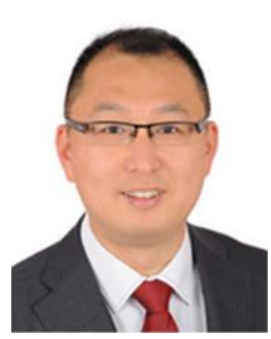

Tao Han was born in Shandong, China, in 1973. He received the Ph.D. degree in instrument science and technology from Shanghai Jiao Tong University, Shanghai, China, in 2002. He was a Visiting Scholar with Tohoku University, Sendai, Japan, in 2003. He is currently a professor at School of Electronic Information and Electrical Engineering in Shanghai Jiao Tong University. His current research interests include acoustic wave devices simulation, wireless surface acoustic wave sensors system, and ultrasound-based measurement. Prof. Han is a Technical Program Committee Member of the IEEE Ultrasonics Symposium.

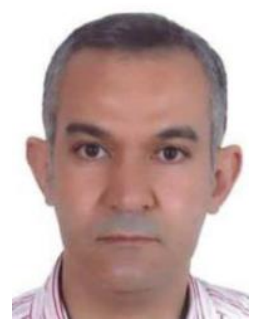

Omar Elmazria (M'2001-SM'16) received his $\mathrm{Ph} . \mathrm{D}$. degree in electronics from Metz University, France, in 1996 and he joined the University of Nancy I as associate Professor of electronic and communication systems in 1997 and as a full Professor in 2003.

$\mathrm{He}$ was the Head of the Micronanosystems group within the Institut Jean Lamour. His current research focus on layered structures based surface acoustic waves (SAW) devices for communication systems and sensing applications.

Pr. Elmazria co-authored more than 150 papers in refereed international journals and in proceeding of international conferences. In 2017, he received the medal of URSI-France (International Union of Radio Science). 